\title{
SMALL AMPLITUDE CAPILLARY-GRAVITY WAVES IN A CHANNEL OF FINITE DEPTH
}

\author{
by M. C. W. JONES
}

(Received 12 November, 1987)

\section{Introduction}

1.1 InTRODUCtory Remarks. Recently a number of studies (Chen \& Saffman [2], Jones \& Toland [7, 11], Hogan [5]) have been made of periodic capillary-gravity waves which form the free surface of an ideal fluid contained in a channel of infinite depth. However, little work appears to have been done on the corresponding problem when the depth is finite. The most significant contributions appear to be those of Reeder \& Shinbrot [9], Barakat \& Houston [1] and Nayfeh [8] all of whom confined themselves to Wilton ripples (see \$1.3). Yet there are sound reasons why such a study should be made. For quite apart from the unsolved problem regarding the type of capillary-gravity waves which may occur at finite depths, the consideration of the finite depth problem may be regarded as a first step in the study of solitary capillary-gravity waves. In this paper, a new integral equation for the infinite depth problem, due to J. F. Toland and the author, is adapted to be of use in tackling the finite depth problem. Using this we obtain results for the exact equations of motion which answer rigorously the questions of existence and multiplicity of small amplitude solutions of the periodic capillary-gravity wave problem of finite depth.

1.2 The PROBlem AND tHe main Results. We shall be considering steady, twodimensional, symmetric waves of period $2 \pi / k$ which arise as the free surface of an ideal fluid of mean depth $h$ contained in a channel of infinite extent with a horizontal bottom. The forces on the fluid are those of gravity $g$ (assumed constant throughout) and surface tension $T$. The phase speed of the waves will be denoted by $c$.

It is obvious that for any values of $h, T$ and $c$, one possible motion is uniform horizontal laminar flow with flat free surface. We shall investigate the possibility of the free surface comprising a uniform horizontal wave train of small amplitude waves. Specifically we shall determine at what values of the phase speed a branch of waves may bifurcate from the uniform horizontal flow. Suppose first that $T$ and $h$ are fixed. Then it is a consequence of the classical theory of bifurcation [3] that a necessary condition for bifurcation to take place from a phase speed $c$ is that the problem linearized about the horizontal flow should have a non-trivial solution. Such a value of $c$ is called an eigenspeed. We shall see that there is a countably infinite set of isolated eigenspeeds $\left\{c_{n}\right\}$. Further, we shall show that if $\frac{T}{g h^{2}} \geq \frac{1}{3}$ (i.e. for all sufficiently small depths), then every eigenspeed has one dimensional solution space, whatever the value of $k$ is. (Such an eigenspeed will be termed a simple eigenspeed.) The classical theory [3] then yields the result that a single curve of small amplitude waves bifurcates from the horizontal flow at

Glasgow Math. J. 31 (1989) 141-160. 
each eigenspeed. Hence in a neighbourhood of any eigenspeed only one possible non-trivial wave can occur.

A much more interesting case is that when $\frac{T}{g h^{2}}<\frac{1}{3}$. When this is the case, we shall show that for any eigenspeed $c_{n}$, there is always a value of the wavenumber $k$ such that the linearized problem has a two dimensional solution space. (Physically this means that two harmonics of the wave are travelling with the same phase speed.) Such an eignespeed will be termed a double eigenspeed. The question of whether bifurcation occurs from a double eignespeed is not one which is covered by the classical theory but we shall be able to show that bifurcation does indeed occur and in fact more than one branch of small amplitude waves bifurcates from the horizontal flow at $c_{n}$. Hence for certain values of the phase speed close to $c_{n}$ there may exist more than one capillary-gravity wave. Precisely how many different waves are possible depends on whether the solution space of the linearized equation contains the fundamental harmonic or not. Full results are given in $\$ 3$.

Having determined the solution set near a double eigenspeed we shall then examine the effect on this set of a perturbation in the surface tension $T$. It will be found that such a perturbation causes the double eigenspeed to split into two simple eigenspeeds from each of which a single solution curve bifurcates. However one of these solution curves may undergo a further splitting (or secondary bifurcation) corresponding to an increase in the period of the waves.

Throughout all this analysis the mean depth $h$ appears through its hyperbolic tangent $\tanh h$. For "small" depths i.e. those for which $\tanh h \sim h$, it is possible to approximate $\tanh h$ by the first few terms in its Taylor series and analytic results are obtainable. The case $\tanh h \sim 1$ corresponds to infinite depth, while the remaining case, i.e. that when $\tanh h$ is neither close to $h$ nor unity, must be studied by numerical means.

1.3 RELATED wORK. Work on capillary-gravity waves in a channel of finite depth has been carried out by Barakat \& Houston [1], Nayfeh [8] and also, in a paper largely concerned with solitary waves, Hunter \& Vanden-Broeck [6]. The methods of these authors were largely formal but they identified the eigenspeeds and calculated the first few terms in the power series expansion of the waves along the bifurcating branches. However, they only treated the case in which the wave interacts with its first harmonic (the waves which arise here are called Wilton ripples) and as we shall see this case is not typical. This paper contains a rigorous analysis of the case in which two adjacent harmonics of the wave interact with each other. Hence our work generalises that of these authors and also yields a vindication of the results obtained by their formal approach. Reeder \& Shinbrot [9] analysed the problem with more rigour but again they confined themselves to Wilton ripples. Capillary-gravity waves on deep water have been treated formally by Chen \& Saffman [2] and rigorously by Jones \& Toland $[\mathbf{7}, 11]$.

The more mathematical aspects of the problem considered here, when a double eigenvalue splits, under perturbation, into two simple ones have been studied by Shearer $[10]$ and also by Golubitsky \& Schaeffer [4]. 


\section{The problem}

2.1 The Physical PROBlem. We shall be considering steady, two-dimensional, periodic and symmetric waves which form the free surface of an ideal fluid which is contained in a horizontal channel of finite depth and infinite horizontal extent. We shall denote the wave number by $k$, so that the wavelength is $\frac{2 \pi}{k}$. (The reason for defining the wavelength in terms of the wave number rather than vice-versa will become clear shortly.) The forces on the fluid are those of gravity $g$ (assumed constant throughout) and surface tension $T$.

This situation can be formulated mathematically as a free boundary problem as follows. One period of the flow may be identified with the region $S$ in the complex plane

$$
S=\{x+i y \mid-\pi / k<x<\pi / k, \quad 0<y<H(x)\},
$$

where $H(x)$ is a smooth even function of period $2 \pi / k$. (Of course $H(x)$ is not known a-priori). The curve

$$
\Pi=\{x+i H(x) \mid-\pi / k<x<\pi / k\}
$$

is called the free surface. Since the motion is irrotational, we can define the complex potential in $S, w(z)=\phi(z)+i \psi(z)$ which is related to the velocity $(u(z), v(z)), z \in S$ by

$$
-\frac{d w}{d z}=-\psi_{y}-i \psi_{x}=-\phi_{x}+i \phi_{y}
$$

Then the phase speed $c$ is defined by

$$
c=\frac{\left(\phi\left(\frac{\pi}{k}\right)-\phi\left(-\frac{\pi}{k}\right)\right) k}{2 \pi} .
$$

Since the free surface $\Pi$ and the line $y=0$ are both streamlines, $\psi$ is constant along them. It is no loss of generality to assume

$$
\psi(z)=0, \quad z \in \Pi
$$

and the mean depth $h$ is defined as

$$
h=-c^{-1} \psi(x)
$$

Because of symmetry we have

$$
\phi_{y}\left( \pm \frac{\pi}{k}+i y\right)=0
$$

and finally it follows from Bernoulli's theorem applied along $\Pi$ that

$$
\frac{1}{2}|\nabla \psi(x+i H(x))|^{2}+g H(x)-T \frac{H^{\prime \prime}(x)}{\left(1+H^{\prime}(x)^{2}\right)^{3 / 2}} \quad \text { is const. , } x \in\left[-\frac{\pi}{k}, \frac{\pi}{k}\right] .
$$


Then by the existence of a solution to the periodic capillary-gravity wave problem with mean depth $h$, phase speed $c$ and surface tension $T$, it is meant that there exist a number $k$ and functions $H(x)$ and $w(z)$ which satisfy conditions (2.1) to (2.6).

2.2 The integral equation. The free boundary value problem (2.1) to (2.6) will be studied by transforming it into an integral equation for $\theta$, the angle between the free surface of the wave and the horizontal. First we shall recall a few facts concerning Fourier series. Let $h$ and $k$ be any positive numbers. Then if $\theta$ is a continuously differentiable, odd function of period $\frac{2 \pi}{k}, \theta$ may be expanded in a Fourier series as

$$
\theta(s) \sim \sum_{l=1}^{\infty} A_{l} \sin k l s
$$

where $\left\{A_{l}\right\}$ is the sequence of Fourier coefficients. Then we shall define $\tau(s)$ to be the function whose Fourier series is

$$
A_{0}+\sum_{l=1}^{\infty} A_{l} \operatorname{coth} k l h \cos k l s
$$

where $A_{0}$ satisfies

$$
e^{A_{0}} \int_{-\pi / k}^{\pi / k} \exp \left(\sum_{l=1}^{\infty} \frac{A_{l} \cos k l s}{\sinh k l h}\right) d s=\frac{2 \pi}{k} .
$$

It may be proved using Privalov's theorem [12], that (2.7) is the Fourier series of a Hölder continuous function.

We can now prove the following theorem which provides a precise link between solutions of a certain equation and the existence of capillary-gravity waves.

THEOREM 1. Suppose $h$ and $k$ are positive numbers, and $\theta$ is a continuously differentiable, real-valued, odd function with period $2 \pi / k$ which satisfies $|\theta(s)|<$ $\frac{\pi}{2}$ for all s. Suppose in addition there are positive numbers $T$ and $c$ for which $\theta$ and $\tau$ (where $\tau$ is defined as above) satisfy the equation

$$
\begin{aligned}
T \theta(s)= & \left(\int_{0}^{\pi / k} \exp \tau(t) d t\right)^{-1}\left(c^{2} \int_{0}^{s} \int_{0}^{\pi / k} \sinh (\tau(x)-\tau(t)) d t d x\right. \\
& \left.+g \int_{0}^{s} \int_{0}^{\pi / k} \int_{y}^{x} \exp \{\tau(x)+\tau(y)+\tau(t)\} \sin \theta(t) d t d y d x\right) .
\end{aligned}
$$

Then there exists a solution to the periodic capillary-gravity wave problem of period $2 \pi / k$, mean depth $h$, phase speed $c$ and surface tension $T$. 
Moreover there is a parameterisation of the free surface $\{(x(s), y(s)): s \in$ $\left.\left[-\frac{\pi}{k}, \frac{\pi}{k}\right]\right\}$ such that $-\theta(s)$ is the angle between the wave profile and the horizontal at the point $(x(s), y(s))$.

Proof. Let $R=\left\{w=s+i u: \frac{-\pi}{k}<s<\frac{\pi}{k},-h<u<0\right\}$. Now define $\tilde{\theta}(w)$ to be the function such that $\bar{\theta}(s+i 0)=\theta(s), \tilde{\theta}(w)=0$ on the rest of $\partial R$ and $\tilde{\theta}(w)$ is harmonic in $R$. Now let $\tilde{\tau}(w)$ be the unique function such that $\tilde{\tau}(w)-i \tilde{\theta}(w)$ is analytic in $R$ and which satisfies

$$
\int_{-\pi / k}^{+\pi / k} \exp \tilde{\tau}(s-i h) d s=\frac{2 \pi}{k}
$$

Then it is clear that

$$
\bar{\theta}(w)=\sum_{l=1}^{\infty} A_{l} \frac{\sinh k l(u+h)}{\sinh k l h} \sin k l s,
$$

and an elementary calculation using the Cauchy-Riemann equations yields that

$$
\tilde{\tau}(w)=A_{0}+\sum_{l=1}^{\infty} A_{l} \frac{\cosh k l(u+h)}{\sinh k l h} \cos k l s
$$

where $A_{0}$ is chosen so that $(2.8)$ is satisfied. Then $\tilde{\tau}(s+i 0)$ is the function whose Fourier series is $A_{0}+\sum_{i=1}^{\infty} A_{l} \operatorname{coth} k l h \cos k l s$ and so by $(2.7) \tilde{\tau}(s+i 0)=\tau(s)$.

Now define a function $m$ on $R$ by putting

$$
m(w)=\int_{-i h}^{w} \exp (\tilde{\tau}(\zeta)-i \bar{\theta}(\zeta)) d \zeta
$$

Now since $|\bar{\theta}|<\frac{\pi}{2}$ on $\partial R$ it follows from the maximum principle that $|\bar{\theta}|<\frac{\pi}{2}$ in $R$. This fact enables us to prove $m$ is injective. For if there were distinct $w_{1}$ and $w_{2}$ such that $m\left(w_{1}\right)=m\left(w_{2}\right)$, this would mean

$$
\int_{\omega_{1}}^{\omega_{2}} \exp (\tilde{\tau}(\zeta)) \cos \tilde{\theta}(\zeta) d \zeta=0
$$

which contradicts the fact that $|\tilde{\theta}|<\frac{\pi}{2}$. In addition $m^{\prime}(w) \neq 0$ in $R$, so $m$ is a conformal mapping from $R$ onto some region $S$ of the complex $z$-plane.

It further follows from (2.9) and $(2.10)$ that $\bar{\theta}(w)=-\bar{\theta}(-\bar{w})$ and $\tilde{\tau}(w)=\tilde{\tau}(-\bar{w})$. It is then straightforward to show that $S$ is bounded by the lines $x= \pm \frac{\pi}{k}, y=0$ and the curve 
$\Gamma=\left\{x+i H(x): x \in\left[\frac{-\pi}{k}, \frac{\pi}{k}\right]\right\}$ where $H$ is a smooth, even function of period $\frac{2 \pi}{k}$. Therefore

$$
x+i H(x)=m(s+i 0), \quad s \in\left[-\frac{\pi}{k}, \frac{\pi}{k}\right]
$$

and $\Gamma$ is given parametrically by

$$
(x(s), y(s))=\left(\int_{0}^{s} e^{\tau(t)} \sin \theta(t) d t, e^{A_{0}} \int_{-h}^{0} \exp \left(\sum_{l=1}^{\infty} \frac{A_{l} \cosh k l(t+h)}{\sinh k l h}\right) d t-\int_{0}^{s} e^{\tau(t)} \cos \theta(t) d t\right) .
$$

Now define a complex potential on $S$ by putting

$$
w(z)=\phi(z)+i \psi(z)=c m^{-1}(z) .
$$

We shall then show that all the conditions defining a flow are satisfied. First note that

$$
\begin{aligned}
-\frac{d w}{d z} & =u(z)-i v(z) \\
& =-\psi_{y}-i \psi_{x}=\frac{c}{m^{\prime}\left(m^{-1}(z)\right)} \\
& =c \exp \left(-\bar{\tau}\left(m^{-1}(z)\right)+i \bar{\theta}\left(m^{-1}(z)\right)\right),
\end{aligned}
$$

and hence

$$
u^{2}(z)+v^{2}(z)=|\nabla \psi(z)|^{2}=c^{2} \exp \left(-2 \tilde{\tau}\left(m^{-1}(z)\right)\right) .
$$

Thus at any point $z \in S$, the speed of the flow is $c \exp \left(-\tilde{\tau}\left(m^{-1}(z)\right)\right)$ and the angle of the direction of the flow to the horizontal is $-\tilde{\theta}\left(m^{-1}(z)\right)$. It is then a straightforward exercise using (2.14) and the definition of the mapping $m$ to show that conditions (2.2) to (2.5) are satisfied. It remains to verify (2.6). If we differentiate $(\mathcal{W})$ and rearrange it we can obtain that

$$
\frac{c^{2}}{2} e^{-2 \tau(s)}-g \int_{0}^{s} e^{\tau(t)} \sin \theta(t) d t+T e^{-\tau(s)} \theta^{\prime}(s)=\text { const. } s \in\left[-\frac{\pi}{k}, \frac{\pi}{k}\right] .
$$

Now it follows from (2.12) that $H^{\prime}(x)=-\tan \theta(s)$ and a calculation yields that

$$
H^{\prime \prime}(x)=-e^{-\tau(s)} \cos ^{-3} \theta(s) \theta^{\prime}(s) .
$$

Now on substituting (2.12) and (2.16) into (2.6) there results

$$
\frac{c^{2}}{2} e^{-2 \tau(s)}-g \int_{0}^{s} e^{\tau(t)} \sin \theta(t) d t+T e^{-\tau(s)} \theta^{\prime}(s)
$$

which is constant by (2.17). 
2.3 Symmetries. $(\mathcal{W})$ will be written in abbreviated form as

$$
T \theta=F(c, \theta) \text {. }
$$

(We suppress the dependence on $g$ since this is regarded as an absolute constant.) It is important to realise that mathematically distinct solutions $\theta$ of $(W)$ need not correspond to physically distinct capillary-gravity waves. The following results, concerning the invariance of $F(c, \cdot)$ enable us to identify different solutions $\theta$ with the same capillary-gravity wave.

First we need to define some function spaces. Let $C^{1}$ be the Banach space of real-valued continuously differentiable functions on $\mathbb{R}$. For $N \in \mathbb{N}$, define

$$
Z_{N}=\left\{v \in C^{1}: v(-s)=-v(s)=-v\left(s+\frac{2 \pi}{k N}\right), s \in \mathbb{R}\right\},
$$

clearly $Z_{N} \subset C^{1}$. If $v \in Z_{N}$, define $T_{N}: Z_{N} \rightarrow Z_{N}$ by

$$
T_{N}(v(t))=v\left(t+\frac{\pi}{k N}\right)
$$

Then is has been proved [11] that

$$
F\left(\cdot, Z_{N}\right) \subset Z_{N}, \quad \forall N \in \mathbb{N}
$$

and also that

$$
T_{N} F(c, \theta)=F\left(c, T_{N} \theta\right), \quad \theta \in Z_{N} .
$$

In general $\theta \neq T_{N} \theta$, but nevertheless it is a consequence of the symmetries in the problem that $\theta$ and $T_{N} \theta$ correspond to the same capillary-gravity wave, the only difference being a shift of origin in the parameterisation (2.13). For this reason the different families of solutions of $(\mathscr{W})$ which are obtained in what follows do not all correspond to distinct families of capillary-gravity waves.

2.4 BifURCATION THEORY. It is clear that for any fixed values of $T$ and $h$ there is a line of solutions of $(W)$

$$
\Gamma_{T}=\{(c, 0) \mid c \in \mathbb{R}\}
$$

corresponding to uniform horizontal laminar flow with flat free surface. These will be termed the trivial solutions. What we shall attempt to do is to discover for which solutions $(c, 0)$ a continuous curve of non-trivial solutions branches off from $\Gamma_{T}$. Such trivial solutions are called bifurcation points. The definition is

Definition. Suppose that for fixed $T$ there is a curve of solutions

$$
\mathscr{C}=\{(c(t), \theta(t)): t \in(-\delta, \delta)\} \subseteq \mathbb{R} \times Z_{1}
$$

of $(W)$ and that $\theta(t)=0$ if and only if $t=0$. Then $(c(0), 0)$ is called a primary bifurcation point and $\mathscr{C}$ a primary bifurcation branch or curve. 
REMARK. A secondary bifurcation point will be defined in $\$ 3$. When there is no risk of confusion a primary bifurcation point will be referred to simply as a bifurcation point. The first step in finding the bifurcation points is to write $F(c, \theta)$ as

$$
c^{2} L_{1} \theta+g L_{2} \theta+H(c, \theta) .
$$

Here $L_{1}$ and $L_{2}$ are linear functions of $\theta$ given by

$$
\begin{gathered}
L_{1} \theta=\int_{0}^{s} \tau_{1}(x) d x \\
L_{2} \theta=\frac{k}{\pi} \int_{0}^{s} \int_{0}^{\pi / k} \int_{y}^{x} \theta(w) d w d y d x
\end{gathered}
$$

where

$$
\tau_{1}(x)=\sum_{l=1}^{\infty} A_{l} \operatorname{coth} k l h \cos k l x,
$$

and

$$
H(c, \theta)=0\left(\|\theta\|^{2}\right) \text { as }\|\theta\| \rightarrow 0 .
$$

Then it follows from the classical theory of bifurcation [3] that a necessary condition for $\left(c_{0}, 0\right)$ to be a bifurcation point is that there should exist a non-zero $\theta_{0}$ such that

$$
T \theta_{0}=c_{0}^{2} L_{1} \theta_{0}+g L_{2} \theta_{0} .
$$

Such values of $\theta_{0}$ and $c_{0}$ will be termed eigenfunctions and eigenspeeds respectively. This is not in general a sufficient condition for bifurcation to occur. However it again follows from the classical theory that if the solution space of $(\mathscr{L})$ corresponding to $c_{0}$ is one dimensional (such a $c_{0}$ will be termed a simple eigenspeed) then $\left(c_{0}, 0\right)$ is indeed a bifurcation point and a single curve of solutions bifurcates from $\Gamma_{T}$ there. It is an elementary calculation to verify that there is a countably infinite set of positive eigenspeeds $\left\{c_{n}\right\}$, given by

$$
c_{n}^{2}=\left(g n^{-1} k^{-1}+T n k\right) \tanh n k h, \quad n \in \mathbb{N},
$$

and that the eigenfunction corresponding to $c_{n}$ is $\sin n k s$. We shall be concerned with the possibility that for some $N, c_{N}=c_{N+1}$. Then there are two linearly independent eigenfunctions corresponding to $c_{N}$, namely $\sin N k s$ and $\sin (N+1) k s$. The classical theory of bifurcation does not apply and to determine whether or not bifurcation does occur we must employ the method of Lyapunov-Schmidt, described in $\$ 3$. (We could study the more general case in which for some $M, N, c_{M}=c_{N}$ but the algebra would seem too complicated to make this worthwhile. The case considered here is physically important because it means that the double eigenspeed $c_{n}$ is the smallest eigenspeed.) First we shall see under what conditions the set $\left\{c_{n}\right\}$ can contain a double eigenspeed.

To begin with, observe that $\left\{c_{n}^{2}\right\}$ may be described as the set of values of the function

$$
f(x)=\left(g x^{-1} k^{-1}+T x k\right) \tanh x k h
$$




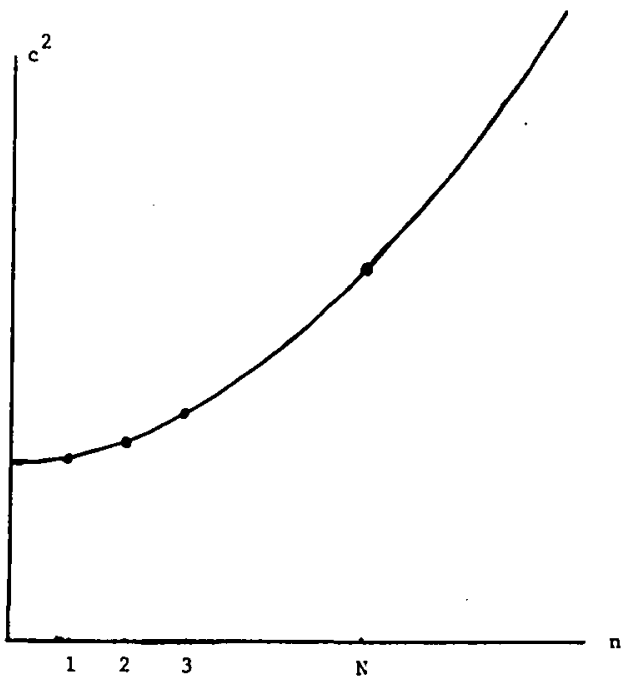

Figure 1. Position of the squares of the eigenspeeds when $\frac{T}{g h^{2}} \geq \frac{1}{3}$.

at positive integer points. Now, it is an easy exercise to verify that if $\frac{T}{g h^{2}} \geq \frac{1}{3}$, then for any vaue of $k, f(x)$ is, for $x>0$, a monotonic increasing function which is unbounded above, (see Fig. 1). Hence for all $n \geq 1, c_{n+1}>c_{n}$, or in other words if $\frac{T}{g h^{2}} \geq \frac{1}{3}$ then for all wavenumbers $k$, the set $\left\{c_{n}\right\}$ consists entirely of simple eigenvalues. Now suppose $\frac{T}{g h^{2}}<\frac{1}{3}$. Then $f^{\prime}(0)=0, f^{\prime \prime}(0)<0$ and $f(x) \rightarrow \infty$ as $x \rightarrow \infty$. Therefore, for $x>0$, the graph of $f$ has a single stationary point which must be a local minimum, (see Fig. 2). We shall show that for any $N \in \mathbb{N}$ it is always possible to choose a value of the wavenumber $k$ such that $c_{N}$ is a double eigenspeed. To see this, just observe that it is required to find a real value of $k$ such that $c_{N}=c_{N+1}$ i.e. such that

$$
\left(g N^{-1} k^{-1}+T N k\right) \tanh N k h=\left(g(N+1)^{-1} k^{-1}+T(N+1) k\right) \tanh (N+1) k h,
$$

or, on rearranging,

$$
\frac{T}{g h^{2}}=\frac{N \tanh (N+1) k h-(N+1) \tanh N k h}{N(N+1) k^{2} h^{2}(N \tanh N k h-(N+1) \tanh (N+1) k h)} \equiv F(k) .
$$

Now, $\lim _{k \rightarrow 0} F(k)=\frac{1}{3}, \lim _{k \rightarrow \infty} F(k)=0$, so there does indeed exist a real $k$ such that (2.18) is satisfied. Put another way, if $\frac{T}{g h^{2}}<\frac{1}{3}$, for any given $N$, there is always a wavenumber $k \in \mathbb{R}$ such that $c_{N}$ is a double eigenspeed. This situation is depicted in Fig. 2. 


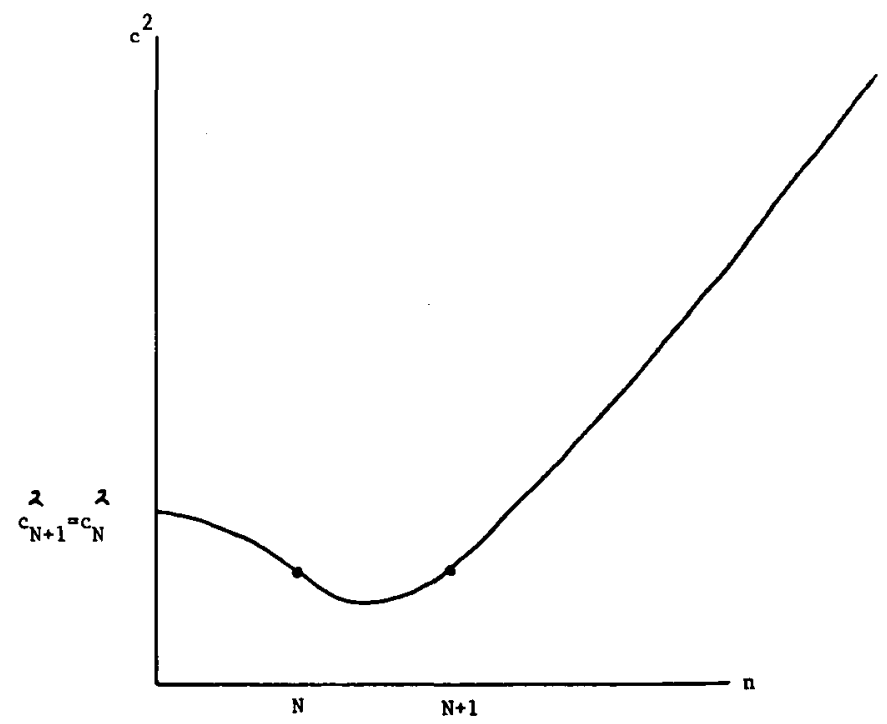

Figure 2. Position of the squares of the eignespeeds when $\frac{T}{g h^{2}}<\frac{1}{3}$.

Remark. The conditions presented here which ensure that all eigenspeeds of the linearized problem are simple, are not directly comparable with those given on p. 402 of [11]. This is because [11] is exclusively concerned with waves of wavelength $2 \pi$ (i.e. $k=1$ ) whereas this paper treats the more general case.

A detailed analysis of the bounds for simple eigenvalues will appear elsewhere.

The rest of the paper will be concerned with determining the solution set of $(\mathscr{W})$ in a neighbourhood of the double eigenspeed $c_{N}$. First we shall make some simplifications and fix some notation. In particular we shall henceforth take $k=1$. This will simplify the algebra considerably and may be done without loss of generality since it just means that the wavenumber $k$ is taken to be the unit of length. Also we shall write, for any $n \in \mathbb{N}$, $\operatorname{th}(n)$ and $\operatorname{cth}(n)$ for tanh $n h$ and coth $n h$ respectively. Now, let

$$
T_{N}=\frac{g(N \operatorname{th}(N+1)-(N+1) \operatorname{th}(N))}{N(N+1)(N \operatorname{th}(N)-(N+1) \operatorname{th}(N+1))}
$$

so that the corresponding double eigenspeed is

$$
c_{N}^{2}=\frac{g(2 N+1) \operatorname{th}(N)) \operatorname{th}(N+1)}{N(N+1)((N+1) \operatorname{th}(N+1)-N \operatorname{th}(N))},
$$

with eigenfunctions $\sin N s$ and $\sin (N+1) s$. We therefore wish to determine the solution set of

$$
T \theta=F(c, \theta)
$$


when $T$ and $c$ are close to $T_{N}$ and $c_{N}$ and $\theta$ is close to zero. What we shall actually do is fix $T$ at $T_{N}$ and study the solution set of $(W)$ in a neighbourhood of $\left(c_{N}, 0\right)$. Then we shall investigate the effect on this solution set of pertrubing $T$ from $T_{N}$.

2.5 THE Reduction of $(W)$. In this section we shall describe the method of Lyapunov-Schmidt, whereby the infinite dimensional problem $(W)$ may be reduced to one of finite dimensions.

Define

$$
\xi=s p\{\sin N s, \sin (N+1) s\},
$$

so that $\xi$ is the solution space of

$$
\begin{gathered}
T_{N} \theta=c_{N}^{2} L_{1} \theta+g L_{2} \theta, \quad \text { and set } \\
\mathscr{F}=\left\{u \in Z_{1}: \int_{0}^{\pi} u(s) \sin n s d s=0, n \in\{N, N+1\}\right\}
\end{gathered}
$$

so that $Z_{1}=\xi \oplus \mathscr{F}$. If $\theta \in Z_{1}$, then

$$
\theta=x \sin N s+y \sin (N+1) s+f
$$

where $f \in \mathscr{F}$ and $(x, y) \in \mathbb{R}^{2}$ (sometimes we shall use this to identify $\mathbb{R}^{2}$ with $\xi$ ). Also, let $P$ and $Q$ be the projections of $Z_{1}$ onto $\xi$ and $\mathscr{F}$ respectively. Then if we introduce new variables $\alpha$ and $\beta$ by writing

$$
c^{2}=c_{N}^{2}+\alpha g, \quad T=T_{N}+\beta g .
$$

$(W)$ then takes the form

$$
\left(T_{N}+\beta g\right)(x \sin N s+y \sin (N+1) s+f)=F\left(c_{N}+\alpha g, x \sin N s+y \sin (N+1) s+f\right)
$$

which is equivalent to the two equations

$$
\begin{gathered}
\left(T_{N}+\beta g\right)(x \sin N s+y \sin (N+1) s)=P F\left(c_{N}+\alpha g, x \sin N s+y \sin (N+1) s+f\right) \\
\left(T_{N}+\beta g\right) f=Q F\left(c_{N}+\alpha g, x \sin N s+y \sin (N+1) s+f\right) .
\end{gathered}
$$

It now follows from the Implicit Function Theorem that there is a neighbourhood $U$ of the origin in $\mathbb{R}^{4}$ and a function $z: U \rightarrow F$ such that $f=z(\alpha, \beta, x, y)$ is the unique solution of (2.21b) for $(\alpha, \beta, x, y) \in U$. If we substitute $f=z(\alpha, \beta, x, y)$ in (2.21a) we obtain

$$
\begin{aligned}
\beta g(x \sin N s+y \sin (N+1) s)= & P F\left(c_{N}+\alpha g, x \sin N s+y \sin (N+1) s+z(\alpha, \beta, x, y)\right) \\
& -T_{n}(x \sin N s+y \sin (N+1) s) .
\end{aligned}
$$

This is known as the bifurcation equation. It is a pair of polynomial equations in the four variables $(\alpha, \beta, x, y)$ and will be written in the abbreviated form

$$
\begin{aligned}
& \beta x=f_{1}(\alpha, \beta, x, y) \\
& \beta y=f_{2}(\alpha, \beta, x, y) .
\end{aligned}
$$


$(\mathscr{B})$ is equivalent to the infinite dimensional problem $(\mathscr{W})$ in the sense that to every solution $(\alpha, \beta, x, y)$ of $(\mathscr{B})$ there coresponds a solution $(\alpha, \beta, x \sin N s+y \sin (N+1) s+$ $z(\alpha, \beta, x, y))$ of $(\mathscr{W})$. The polynomials $f_{1}$ and $f_{2}$ may, in principle, be computed up to any degree of accuracy but due to the rapidly increasing labour involved, only the terms up to the third degree are calculated. We do not give the details of these calculations but they are along the same lines as those to be found in [11] for the case of infinite depth. What we wish to do now is provide a description of the solution set of $(\mathscr{B})$ for $(\alpha, \beta, x, y)$ in a neighbourhood of the origin in $\mathbb{R}^{4}$. First we shall fix $\beta$ at zero. Then the only possible bifurcation point in a neighbourhood of the origin is at $\alpha=0$, and we shall determine the number and nature of the solution curves which bifurcation from this point. Next we shall perturb $\beta$ from zero. Now there are two possible bifurcation points near the origin: at $\alpha=N \beta \operatorname{th}(N)$ and $\alpha=(N+1) \beta \operatorname{th}(N+1)$. Since the linearized problem has one dimensional solution space at each of these points it follows from the classical theory that a single curve of solutions does indeed bifurcate from each of these points. However we shall describe the nature of these curves and also determine whether secondary bifurcation occurs along them. The precise form of $f_{1}$ and $f_{2}$ will be given in due course, but first we shall give some properties of the bifurcation equations which arise from the symmetry observations of $\S 2.3$. Recall that when $N>1, Z_{N}$ is a proper subspace of $Z_{1}$, $F\left(\cdot, Z_{N}\right) \subseteq Z_{N}$, and further that when $\alpha=N \beta \operatorname{th}(N)$, the solution space of $(\mathscr{L})$ is one dimensional and spanned by $\sin N s \in Z_{N}$. Then it is a consequence of the invariance results of $\$ 2.3$ that $z(\alpha, \beta, x, 0) \in Z_{N}$ and hence it follows from the definition of $f_{2}$ and the fact that $\sin (N+1) s$ is orthogonal (in the $L_{2}$-sense) to every element of $Z_{N}$ that

$$
f_{2}(\alpha, \beta, x, 0)=0, N>1 \text {. }
$$

Similarly

$$
f_{1}(\alpha, \beta, 0, y)=0, \quad N \in \mathbb{N} .
$$

However, if $N=1$, the argument used to prove (2.22) breaks down. Indeed (2.22) is false in the case $N=1$ and the bifurcation diagrams are different in the cases $N>1$ and $N=1$.

\section{The main results}

In this section we describe the solution set of $(\mathscr{W})$ in a neighbourhood of the origin and identify the primary and secondary bifurcation points. The definition of the latter is

Definition. Suppose that $\mathscr{C}$ is a primary solution curve of $(\mathscr{W})$ which bifurcates from $\left(\alpha^{*}, 0\right)$ and that $\mathscr{D}=\{(\tilde{\alpha}(s), \bar{\theta}(s)): s \in(-\delta, \delta)\}$ is another solution curve such that $\mathscr{C} \cap \mathscr{D}=\{(\tilde{\alpha}, \tilde{\theta})\} \subset \mathscr{C} \backslash\left\{\left(\alpha^{*}, 0\right)\right\}$. Then $(\tilde{\alpha}, \tilde{\theta})$ is said to be a secondary bifurcation point and $\mathscr{D}$ is said to be a secondary bifurcation curve.

From now on the cases $N=1, N>1$ will be treated separately.

3.1 WiLton RIPPles-The CASE $\mathbf{N}=1$. The bifurcation equations are

$$
\beta x=\alpha x c t h(1)-\frac{3 x y}{4} \operatorname{cth}(1)+u(\alpha, \beta, x, y)
$$




$$
\beta y=\frac{\alpha y}{2} \operatorname{cth}(2)-\frac{3 x^{2}}{8} \operatorname{cth}(2)+v(\alpha, \beta, x, y),
$$

where $\left.|(u, v)|=\left.O(\mid \alpha, \beta, x, y)\right|^{3}\right)$ as $(\alpha, \beta, x, y) \rightarrow 0$, and, by (2.22)

$$
u(\alpha, \beta, x, y)=x u_{1}(\alpha, \beta, x, y) .
$$

A detailed analysis of such equations can be found in $[4,10]$. The precise details are rather technical and involve various detailed scaling arguments but the conclusion is that for a qualitative description of the solution set of (3.1) in a neighbourhood of the origin in $\mathbb{R}^{4}$ it is sufficient to study the truncated equations 1.e.

$$
\beta x=\alpha x \operatorname{cth}(1)-\frac{3 x y}{4} \operatorname{cth}(1)
$$

and

$$
\beta y=\frac{\alpha y}{2} \operatorname{cth}(2)-\frac{3 x^{2}}{8} \operatorname{cth}(2) .
$$

First note that one solution of (3.2) is $\alpha=2 \beta \operatorname{th}(2), x=0$, which corresponds to the primary bifurcation curve $\mathscr{C}_{\beta}^{2}$ bifurcating from $\Gamma_{\beta}$ at $(2 \beta \operatorname{th}(2), 0)$. Further, it follows from the symmetry results of $\S 2.3$ that $\mathscr{C}_{\beta}^{2} \subseteq \mathbb{R} \times Z_{2}$ and hence the solutions on $\mathscr{C}_{\beta}^{2}$ correspond physically to water-waves of period $\pi$. As it stands (3.2) gives no indication of the direction of bifurcation from $\Gamma_{\beta}$. However, it is possible to carry out a more detailed calculation to determine the cubic terms in (3.1). From these there results that for some $\epsilon$ (independent of $\beta$ )

$$
\mathscr{C}_{\beta}^{2}=\left\{\left(\alpha^{*}(y, \beta), y \sin 2 s+O\left(y^{2}\right)\right):|y|<\epsilon\right\}
$$

where

$$
\begin{aligned}
\alpha^{*}(y, \beta)= & 2 \beta \operatorname{th}(2)-\frac{y^{2} \operatorname{th}(2)}{32}\left(1+\frac{15+4 \sinh ^{2} h-t h^{2}(1)}{\sinh ^{2} h\left(3-t^{2}(1)\right)}+\frac{3\left(3-t h^{2}(1)\right)}{t h^{3}(2) t h^{3}(1)}\right) \\
& +O\left(y^{2}|(y, \beta)|\right) .
\end{aligned}
$$

Hence $\mathscr{C}_{\beta}^{2}$ is a subcritical pitchfork which is divided into two branches by the bifurcation point $(2 \beta \operatorname{th}(2), 0)$. These branches will be denoted ${ }^{ \pm} \mathscr{C}_{\beta}^{2}$ according as $\pm y>0$ and it follows from the symmetry observations of $\$ 2.3$ that both branches correspond to the same set of capillary-gravity waves.

Since $\mathscr{C}_{\beta}^{2}$ accounts for all non-trivial small solutions of (3.2) for which $x=0$, it now suffices to consider equations (3.2a) divided by $x$ and (3.2b). On rearranging there results that these are equivalent to

$$
\begin{gathered}
x^{2}=\frac{16}{9}(\alpha-2 \beta \operatorname{th}(2))(\alpha-\beta \operatorname{th}(1)) \\
y=\frac{4}{3}(\alpha-\beta \operatorname{th}(1)) .
\end{gathered}
$$

When $\beta>0$, these represent a primary curve $\mathscr{C}_{\beta}^{1}$ which bifurcates subcritically from $\Gamma_{\beta}$ 

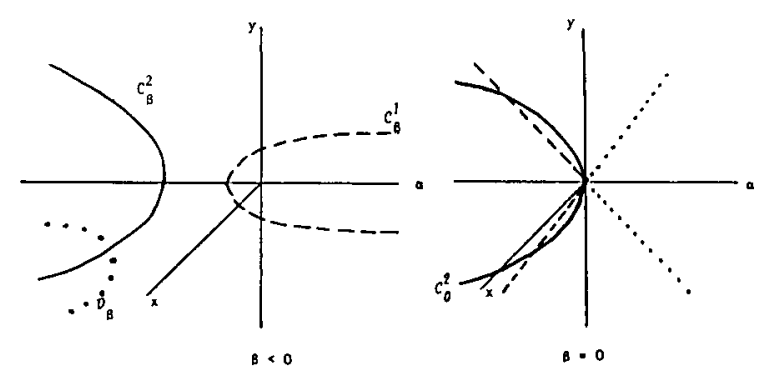

(a)

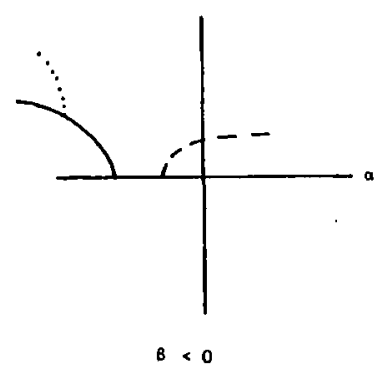

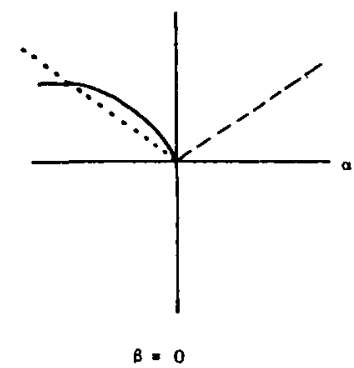

(b)
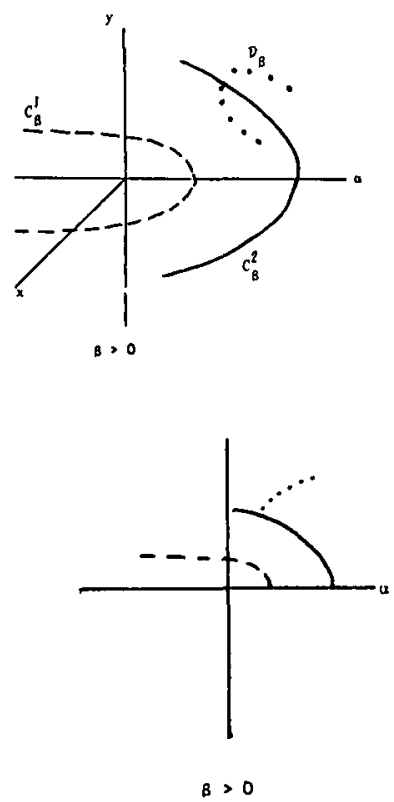

Figure 3. In both these figures $\alpha$ represents the perturbation of the phase speed from $c_{1}$ and $\beta$ the perturbation of the surface tension from $T_{1}$. (a). Small solutions $(\alpha, x, y)$ of the bifurcation equations (3.1) for $\beta$ positive, zero and negative. - represents solutions along which $x=0,----$ and $\cdots$ represent solutions along which neither $x$ nor $y$ is zero. (b). Small solutions $(\alpha, \theta)$ of the capillary-gravity wave problem for $\beta$ positive, zero and negative. - represents waves with minimal period $\pi,---$ and $\cdots$ represents waves with minimal period $2 \pi$.

at $\alpha=\beta \operatorname{th}(1)$, and a secondary curve $\mathscr{D}_{\beta}$ which bifurcates supercritically from $+\mathscr{C}_{\beta}^{2}$ at a point at which $(\alpha, y) \sim\left(2 \beta \operatorname{th}(2), \frac{4}{3} \beta(2 \operatorname{th}(2)-\operatorname{th}(1))\right)$.

When $\beta<0$, the situation is the same except that $\mathscr{C}_{\beta}^{1}$ bifurcates supercritically and $\mathscr{D}_{\beta}$ bifurcates subcritically from $-\mathscr{C}_{\beta}^{2}$. The water-waves corresponding to the solutions on these curves all have minimal period $2 \pi$ and those on the two branches of the same bifurcation curve correspond physically to the same set of water-waves: however the solutions on the different curves $\mathscr{C}_{\beta}^{1}$ and $\mathscr{D}_{\beta}$ are distinct; they cannot be brought into coincidence by a change of origin.

When $\beta=0,(3.5)$ represents two straight lines which intersect at the origin. However, as is suggested by the analysis when $\beta \neq 0$, these are better interpreted as two degenerate pitchfork bifurcations, one super- and one sub-critical which bifurcate from $\Gamma_{0}$ at $\alpha=0$. To every non-zero value of $\alpha$, there are then two solutions $\theta$ of (W) corresponding to the solutions $( \pm x, y)$ of $(3.5)$ and these both correspond to the same water-wave and have minimal period $2 \pi$. (Of course the addition solutions lying on the subcritical curve $\mathscr{C}_{0}^{2}$ are still present.)

The conclusions for all values of $\beta$ are pictured in Fig. 3. Fig. 3(a) depicts the small 
solutions of the bifurcation equations (3.1) while Fig. 3(b) interprets these in the context of solutions of the capillary-gravity wave problem.

3.2 The CASE $N>1$. A long and involved calculation yields that in this case the bifurcation equations are

$$
\begin{gathered}
\beta x=\frac{\alpha x}{N} \operatorname{cth}(N)+A x^{3}+C x y^{2}+p(\alpha, \beta, x, y) \\
\beta y=\frac{\alpha y}{N+1} c t h(N+1)+B y^{3}+D x^{2} y+q(\alpha, \beta, x, y) .
\end{gathered}
$$

The coefficients $A, B, C, D$ are rather complicated functions of $N$ and $h$ and are given in the appendix. The higher order terms satisfy

$$
(p, q)=\left(x p_{1}, y q_{1}\right)
$$

where

$$
\left|p_{1}\right|+\left|q_{1}\right|=O\left(|(\alpha, \beta, x, y)|^{3}\right) \text { as }(\alpha, \beta, x, y) \rightarrow 0 .
$$

For a qualitative analysis of the solutions of (3.6) near the origin, it is sufficient, just as when $N=1$, to study the truncated equations, i.e. (3.6a) and (3.6b) with $p$ and $q$ set to zero. First note that one solution of these equations is

$$
\alpha=N \operatorname{th}(N)\left(\beta-A x^{2}\right), \quad y=0 .
$$

This corresponds to the primary curve $\mathscr{C}_{\beta}^{N}$ which bifurcates from $\Gamma_{\beta}$ at $\alpha=N \beta \operatorname{th}(N)$. (The direction of the bifurcation is dependent on the sign of $A$, which numerical calculations indicate to be positive. This would mean that $\mathscr{C}_{\beta}^{N}$ bifurcatues subcritically.) It follows from the invariance results of $\S 2.3$ that $\mathscr{C}_{\beta}^{N} \subseteq \mathbb{R} \times Z_{N}$. Another solution of the truncated equations is

$$
\alpha=(N+1) \operatorname{th}(N+1)\left(\beta-B y^{2}\right), \quad x=0,
$$

which corresponds to the primary curve $\mathscr{C}_{\beta}^{N+1} \subseteq \mathbb{R} \times Z_{N+1}$ which bifurcates from $\Gamma_{\beta}$ at $\alpha=(N+1) \beta \operatorname{th}(N+1)$. (Again, numerical evidence suggests $B$ is positive which means $\mathscr{C}_{\beta}^{N+1}$ is a subcritical bifurcation.) It further follows [10] that the solutions on $\mathscr{C}_{\beta}^{N} \cup \mathscr{C}_{\beta}^{N+1}$ are the only non-trivial solutions of $(\mathscr{W})$ in a neighbourhood of the origin which belong to $\mathbb{R} \times\left(Z_{N} \cup Z_{N+1}\right)$. In future therefore it suffices to consider equations (3.6) divided by $x$ and $y$ respectively.

Any secondary bifurcation points on $\mathscr{C}_{\beta}^{N} \cup \mathscr{C}_{\beta}^{N+1}$ correspond to solutions of (3.6) for which $x y=0$. A straightforward calculation yields that the only possible such solutions are

$$
\begin{gathered}
x=0, \alpha=\frac{\beta N(N+1)(B-C) \operatorname{th}(N) \operatorname{th}(N+1)}{(N+1) B \operatorname{th}(N+1)-N C \operatorname{th}(N)} \\
y^{2}=\frac{\beta((N+1) \operatorname{th}(N+1)-N \operatorname{th}(N))}{((N+1) B \operatorname{th}(N+1)-N C \operatorname{th}(N))}
\end{gathered}
$$


and

$$
\begin{gathered}
y=0, \alpha=\frac{\beta N(N+1)(D-A) \operatorname{th}(N) \operatorname{th}(N+1)}{(N+1) \operatorname{Dth}(N+1)-N \operatorname{th}(N)} \\
x^{2}=\frac{\beta((N+1) \operatorname{th}(N+1)-N \operatorname{th}(N))}{((N+1) \operatorname{Dth}(N+1)-N \operatorname{tath}(N))} .
\end{gathered}
$$

Now divide the numerator and denominator in the expressions for $x^{2}$ and $y^{2}$ in (3.9) by $N(N+1)$. Then the numerator of both resulting expressions becomes

$$
\beta\left(N^{-1} \operatorname{th}(N+1)-(N+1)^{-1} \operatorname{th}(N)\right),
$$

which has the same sign as $\beta$.

Therefore the existence of solutions of (3.6) which have $x y=0$ is dependent on the signs of

$$
\frac{B}{N} \operatorname{th}(N+1)-\frac{C}{N+1} \operatorname{th}(N)
$$

and

$$
\frac{D}{N} \operatorname{th}(N+1)-\frac{A}{N+1} \operatorname{th}(N)
$$

To determine the signs of these quantities it is necessary to use both analytic and numerical techniques. If we first fix $N$ and let $h \rightarrow \infty$ the limiting value of (3.10) is

$$
\frac{-\left(11 N^{4}+55 N^{3}+39 N^{2}+N+2\right)}{8 N^{2}(N+1)^{3}(N-1)(N+2)}
$$

which is negative for all $N>1$, while that of $(3.11)$ is

$$
\frac{11 N+10}{8 N^{2}(N+1)^{2}}
$$

which is positive for all $N>1$. (It is reassuring to note that these expressions are the same as those obtained in [11] which deal with the case of infinite depth.)

Calculations were carried out on a computer to determine the values of (3.10) and (3.11) for other values of $N$ and $h$. (3.10) and (3.11) were evaluated for integral values of $N$ between 2 and 100 and for $\tanh h$ between $0 \cdot 1$ and 1 at intervals of $0 \cdot 1$. It was found that the signs of (3.10) and (3.11) never changed and indeed for fixed $N,(3.10)$ is a monotonic increasing function of $h$ while (3.11) is a monotonic decreasing function of $h$.

Finally, for "small" values of $N h$ i.e. those for which tanh $N h \sim N h$, it is possible to approximate (3.10) and (3.11) by replacing tanh $N h$ by the first few terms in its Taylor series. If this is done (see appendix) it is seen that the signs of (3.10) and (3.11) are still negative and positive respectively.

The conclusion is therefore that for $\beta>0$ there are two secondary bifurcation points on $\mathscr{C}_{\beta}^{N}$ : one on each of ${ }^{ \pm} \mathscr{C}_{\beta}^{N}$ and none on $\mathscr{C}_{\beta}^{N+1}$. For $\beta<0$, the situation is reversed: there 
is one bifurcation point on each of ${ }^{ \pm} \mathscr{C}_{\beta}^{N+1}$ and none on $\mathscr{C}_{\beta}^{N}$. The two secondary curves are pitchforks since they are given approximately by (3.9). Each is divided into two subsets by the secondary bifurcation point and it is a consequence of the symmetry considerations of $\$ 2.3$ that these four solution curves correspond to only two distinct families of capillary-gravity waves which have minimal period $2 \pi$.

$$
\beta=0
$$

In this case the only possible bifurcation point in a neighbourhood of the origin is at $\alpha=0$ and indeed there are two curves $\mathscr{C}_{0}^{N}$ and $\mathscr{C}_{0}^{N+1}$ bifurcating from this point. To see if there are any others we must seek solutions of (3.6) with $\beta=0$. The truncated form of these equations is easily seen to be equivalent to

$$
\left(\begin{array}{l}
x^{2} \\
y^{2}
\end{array}\right)=\frac{\alpha}{N(N+1) \operatorname{th}(N) \operatorname{th}(N+1)(A B-C D)}\left(\begin{array}{l}
N C \operatorname{th}(N)-(N+1) B \operatorname{th}(N+1) \\
(N+1) \operatorname{Dth}(N+1)-N A \operatorname{th}(N)
\end{array}\right)
$$

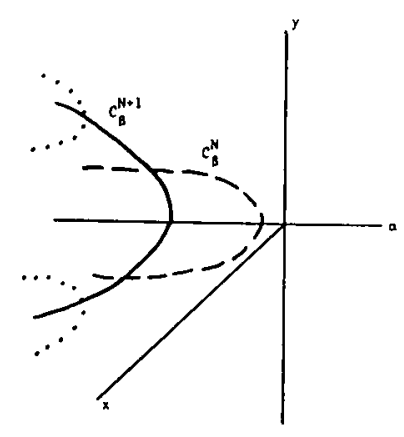

$B<0$

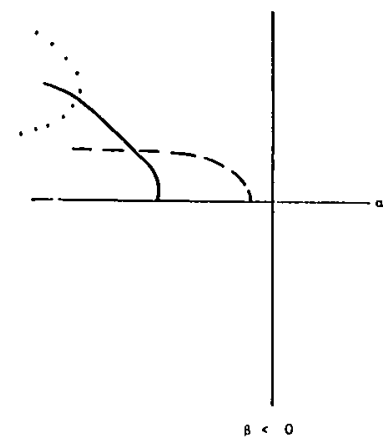

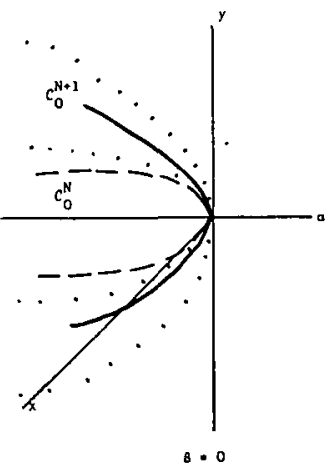

(a)

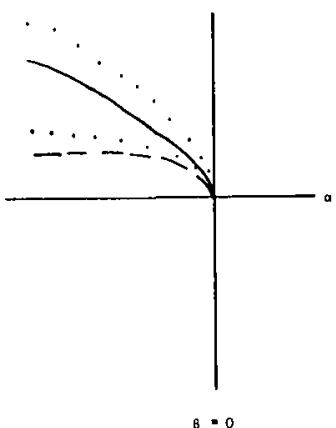

(b)
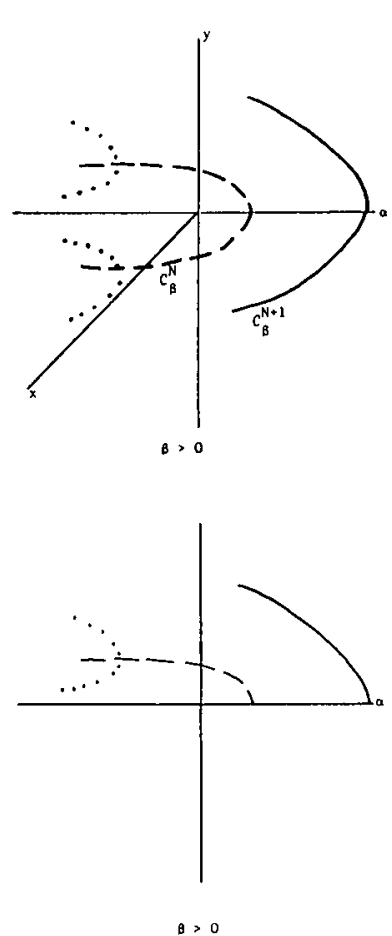

Figure 4. In both these figures $\alpha$ represents the perturbation of the phase speed from $c_{N}$ and $\beta$ the perturbation of the surface tension from $T_{N}, N>1$. (a). Small solutions $(\alpha, x, y)$ of the bifurcation equations (3.6) for $\beta$ positive, zero and negative. - represents solutions along which $x=0,----$ represents solutions along which $y=0, \cdots$ represents solutions along which neither $x$ nor $y$ is zero.(b). Small solutions $(\alpha, \theta)$ of the capillary-gravity wave problem for $\beta$ positive, zero and negative. - represents solutions with minimial period $\frac{2 \pi}{N+1},---$ represents solutions with minimal period $\frac{2 \pi}{N}, \cdots$ represents solutions with minimal period $2 \pi$. 
and the existence of solutions is, as before, dependent on the signs of (3.10) and (3.11). Thus, by the same calculations as when $\beta \neq 0$, there results that two further primary curves bifurcate from $\alpha=0$. These curves are divided by the origin into four subsets and it again follows from symmetry considerations that they correspond to only two distinct families of capillary-gravity waves all of which have minimal period $2 \pi$. (Observe that the sign of $(A B-C D)$ does not influence the existence of solution curves, only their direction. We have not attempted to determine this sign, but as $h \rightarrow \infty$ this quantity tends to the ratio of two polynomials in $N$ which is easily seen to be negative. Hence for sufficiently large depths these bifurcations are subcritical.)

These conclusions are depicted in Fig. 4. Fig. 4(a) shows the small solutions of the bifurcation equations (3.6) while Fig. 4(b) interprets these in the context of capillarygravity waves.

\section{Conclusion}

Broadly speaking the bifurcation diagrams are similar to those obtained in [11] for the case of infinite depth, so it appears that the introduction of the depth as an extra parameter does not affect this problem to any significant extent. It would, however, be possible to obtain triple eigenspeeds for certain critical values of the depth $h$ and it is hoped to present an analysis of this case in due course.

\section{Appendix}

We here present the values of $A, B, C$ and $D$, the coefficients in the bifurcation equations (3.6). Some preliminary definitions are required. Set

$$
\begin{aligned}
& P(N)=(3 N+1)(N-1) \operatorname{th}(N+1) \operatorname{th}(2 N)-3 N(N+1) \operatorname{th}(N) \operatorname{th}(2 N) \\
& +2(2 N+1) \operatorname{th}(N) \operatorname{th}(N+1), \\
& Q(N)=3 N(N+1) \operatorname{th}(N+1) \operatorname{th} 2(N+1)-(3 N+2)(N+2) \operatorname{th}(N) \operatorname{th} 2(N+1) \\
& +2(2 N+1) \operatorname{th}(N) \operatorname{th}(N+1) \text {, } \\
& R(N)=(N+1) \operatorname{th}(N+1)-N \operatorname{th}(N), \\
& S(N)=N^{2}(3 N+2) \operatorname{th}(N+1) \operatorname{th}(2 N+1)-(N+1)^{2}(3 N+1) \operatorname{th}(N) \operatorname{th}(2 N+1) \\
& +(2 N+1)^{2} \operatorname{th}(N) \operatorname{th}(N+1), \\
& T(N)=N^{2}(N+2) \operatorname{th}(N+1) \operatorname{th}(1)-\left(N^{2}-1\right)(N+1) \operatorname{th}(N) \operatorname{th}(1)-(2 N+1) \operatorname{th}(N) \operatorname{th}(N+1), \\
& U(N)=(3 N+1)(N+1) \operatorname{th}(N)+N(3 N+2) \operatorname{th}(N+1), \\
& V(N)=N(N+2) \operatorname{th}(N+1)+\left(1-N^{2}\right) \operatorname{th}(N) .
\end{aligned}
$$


Then

$$
\begin{aligned}
& A=\frac{1}{8 N^{2} t h^{2}(N)}\left(3\left(1-t^{2}(N)\right)+\frac{(2 N+1) t h(N+1)\left(3-2 t h^{2}(N)\right)}{(N+1) R(N)}-\frac{9(N+1) t h(2 N) R(N)}{P(N)}\right), \\
& B=\frac{1}{8(N+1)^{2} t h^{2}(N+1)}\left(3\left(1-t h^{2}(N+1)\right)+\frac{(2 N+1) t h(N)\left(3-2 t h^{2}(N+1)\right)}{N R(N)}\right. \\
& \left.-\frac{9 N \operatorname{th} 2(N+1) R(N)}{Q(N)}\right) \\
& C=\frac{1}{4 N^{2} t^{2}(N+1)}\left(3-t h^{2}(N+1)+\frac{(2 N+1) t h(N+1)\left(2-t h^{2}(N+1)\right)}{(N+1) R(N)}\right. \\
& +\frac{2 N^{2} \operatorname{th}(N+1)-\left(2 N^{2}+8 N+3\right) \operatorname{th}(N)}{(2 N+1) \operatorname{th}(N)}-\frac{N(3 N+2) \operatorname{th}(2 N+1) U(N) R(N)}{(N+1)(2 N+1) \operatorname{th}(N) S(N)} \\
& \left.+\frac{N(N+2) \operatorname{th}(1) V(N) R(N)}{(N+1) \operatorname{th}(N) T(N)}\right) \\
& D=\frac{1}{4(N+1)^{2} t h^{2}(N)}\left(3-t h^{2}(N)+\frac{(2 N+1) t h(N)\left(2-t h^{2}(N)\right)}{N R(N)}\right. \\
& +\frac{\left(2 N^{2}-4 N-3\right) \operatorname{th}(N+1)-2(N+1)^{2} \operatorname{th}(N)}{(2 N+1) \operatorname{th}(N+1)} \\
& \left.-\frac{(N+1)(3 N+1) \operatorname{th}(2 N+1) U(N) R(N)}{N(2 N+1) \operatorname{th}(N+1) S(N)}+\frac{\left(N^{2}+4 N+1\right) \operatorname{th}(1) V(N) R(N)}{N \operatorname{th}(N+1) T(N)}\right) \text {. }
\end{aligned}
$$

When $N h$ is small e.g. $<0 \cdot 1, \tanh N h$ may be approximated by the first few terms in its Taylor expansion i.e. $\tanh N h \sim N h-\frac{N^{3} h^{3}}{3}+\frac{2 N^{5} h^{5}}{15}$, and the signs of (3.10) and (3.11) may be determined analytically: This is quite a complicated calculation since the first non-vanishing term in $P(N), Q(N), S(N)$ and $T(N)$ is that in $h^{6}$. Again we need a preliminary definition to describe these results. Let

$$
F(N)=18 N^{7}+177 N^{6}+680 N^{5}+1334 N^{4}+1416 N^{3}+829 N^{2}+254 N+32 .
$$

Then there results that for small $N h$,

$$
\begin{aligned}
\frac{B}{N} \operatorname{th}(N+1) & -\frac{C}{(N+1)} \operatorname{th}(N) \sim-\frac{135}{4 N^{2}(N+1)^{5}(N+2) h^{5}} \\
& \times\left(\frac{F(N)}{2\left(3 N^{4}+12 N^{3}+23 N^{2}+16 N+4\right)\left(18 N^{3}+27 N^{2}+13 N+2\right)}+\frac{(N+2)}{(N-1)}\right)
\end{aligned}
$$


which is negative, and

$$
\begin{aligned}
\frac{D}{N} \operatorname{th}(N+1)-\frac{A}{N+1} \operatorname{th}(N) & \sim \frac{135}{4 N^{5}(N+1)^{2} h^{5}} \\
\times & \left(\frac{18 N^{5}+57 N^{4}-41 N^{3}-23 N^{2}+7 N+6}{2 N(N+1)\left(9 N^{2}-6 N-1\right)(N-1)(N+2)}+\frac{(3 N+1)}{9 N^{2}+9 N+2}\right)
\end{aligned}
$$

which is positive.

Acknowledgement. It is a pleasure to thank Professor J. F. Toland for his invaluable help and advice during the preparation of this work. I would also like to thank the referee for helpful and constructive criticism. A preliminary version of these results was given at the 1987 SERC symposium on differential equations held in Gregynog, Wales.

\section{REFERENCES}

1. R. Barakat and A. Houston, Nonlinear periodic capillary-gravity waves on a fluid of finite depth, J. Geophys. Res., 73 (1968), 6545-6554.

2. B. Chen and P. G. Saffman, Steady gravity-capillary waves on deep water-I. weakly non-linear waves, Stud. App. Math., 60 (1979), 183-210.

3. M. G. Crandall and P. H. Rabinowitz, Bifurcation from a simple eigenvalue, J. Funct. Anal., 8 (1971), 321-340.

4. M. Golubitsky and D. G. Schaeffer, Singularities and Groups in Bifurcation Theory (I). (Springer-Verlag, 1985).

5. S. J. Hogan, Some effects of surface tension on steep water waves. Parts 1, 2 and 3, J. Fluid Mech., 91 (1979), 167-180; 96 (1980), 417-445; 110 (1981), 381-410.

6. J. K. Hunter and J.-M. Vanden-Broeck, Solitary and periodic gravity waves of finite amplitude, J. Fluid Mech., 134 (1983), 205-219.

7. M. C. W. Jones and J. F. Toland, Symmetry and the bifurcation of capillary-gravity waves, Arch. Rat. Mech. Anal., 96 (1986), 29-53.

8. A. H. Nayfeh, Finite amplitude surface waves in a liquid layer, J. Fluid Mech., 40(4) (1979), 671-684.

9. J. Reeder and M. Shinbrot, On Wilton ripples, I: formal derivation of the phenomenon, Wave motion, 3 (1981), 115-135.

10. M. Shearer, Secondary bifurcation near a double eigenvalue, SIAM J. Math. Anal., 11 (2) (1980), 365-389.

11. J. F. Toland and M. C. W. Jones, The bifurcation and secondary bifurcation of capillary-gravity waves, Proc. $R$. Soc. Lond. A, 399 (1985), 391-417.

12. A. Zymund, Trigonometric series Vol. I (Cambridge University Press, 1959).

Department of Pure Mathematics,

The QueEN'S UNIVERSITY OF BELFAST,

BELFAST BT7 1NN.

Northern Ireland. U. K. 